\title{
OBO-Fused Benzo[fg]tetracene as Acceptor With Potential for Thermally Activated Delayed Fluorescence Emitters
}

\author{
Zhen Zhang ${ }^{1}$, Shiv Kumar ${ }^{2}$, Sergey Bagnich ${ }^{3}$, Eduard Spuling ${ }^{1,2}$, Fabian Hundemer ${ }^{1}$, \\ Martin Nieger ${ }^{4}$, Zahid Hassan ${ }^{1}$, Anna Köhler ${ }^{3 *}$, Eli Zysman-Colman ${ }^{2 *}$ and Stefan Bräse ${ }^{1,5 *}$ \\ ${ }^{1}$ Institute of Organic Chemistry (IOC), Karlsruhe Institute of Technology (KIT), Karlsruhe, Germany, ${ }^{2}$ Organic Semiconductor \\ Centre, EaStCHEM School of Chemistry, University of St Andrews, St Andrews, United Kingdom, ${ }^{3}$ Soft Matter \\ Optoelectronics, Bayreuth Institute for Macromolecular Research (BIMF) \& Bavarian Polymer Institute (BPI), University of \\ Bayreuth, Bayreuth, Germany, ${ }^{4}$ Department of Chemistry, University of Helsinki, Helsinki, Finland, ${ }^{5}$ Institute of Biological and \\ Chemical Systems - Functional Molecular Systems (IBCS-FMS), Karlsruhe Institute of Technology (KIT), \\ Eggenstein-Leopoldshafen, Germany
}

\section{OPEN ACCESS}

Edited by:

Guigen Li,

Texas Tech University, United States

Reviewed by:

Xu-Lin Chen,

Chinese Academy of Sciences, China

Zujin Zhao,

South China University of

Technology, China

*Correspondence:

Anna Köhler

anna.koehler@uni-bayreuth.de

Eli Zysman-Colman

eli.zysman-colman@st-andrews.ac.uk

Stefan Bräse

braese@kit.edu

Specialty section

This article was submitted to Organic Chemistry,

a section of the journal

Frontiers in Chemistry

Received: 18 May 2020

Accepted: 19 August 2020

Published: 30 September 2020

Citation:

Zhang Z, Kumar S, Bagnich S, Spuling E, Hundemer F, Nieger $M$,

Hassan Z, Köhler A,

Zysman-Colman E and Bräse S (2020)

OBO-Fused Benzo[fg]tetracene as Acceptor With Potential for Thermally

Activated Delayed Fluorescence

Emitters. Front. Chem. 8:563411.

doi: 10.3389/fchem.2020.563411
Six luminophores bearing an OBO-fused benzo[fg]tetracene core as an electron acceptor were designed and synthesized. The molecular structures of three molecules (PXZ-OBO, 5PXZ-OBO, 5DMAC-OBO) were determined by single crystal $X$-ray diffraction studies and revealed significant torsion between the donor moieties and the OBO acceptor with dihedral angles between 75.5 and $86.2^{\circ}$. Photophysical studies demonstrate that blue and deep blue emission can be realized with photoluminescence maxima $\left(\lambda_{\mathrm{PL}}\right)$ ranging from 415 to $480 \mathrm{~nm}$ in $\mathrm{mCP}$ films. The emission energy is modulated by simply varying the strength of the donor heterocycle, the number of donors, and their position relative to the acceptor. Although the DMAC derivatives show negligible delayed emission because of their large singlet-triplet excited state energy difference, $\Delta E_{S T}$, PXZ-based molecules, especially PXZ-OBO with an experimental $\Delta E_{\mathrm{ST}}$ of $0.25 \mathrm{eV}$, demonstrate delayed emission in blend $\mathrm{mCP}$ films at room temperature, which suggests triplet exciton harvesting occurs in these samples, potentially by thermally activated delayed fluorescence.

Keywords: boron emitters, fluorescence, OLED, TADF, boron

\section{INTRODUCTION}

As one of the most promising electroluminescent technologies, organic light-emitting diodes (OLEDs) have attracted significant attention and are now being commercialized across a number of different product lines (Endo et al., 2011; Uoyama et al., 2012; Tao et al., 2014; Wong and Zysman-Colman, 2017; Yang et al., 2017). State-of-the-art phosphorescent OLEDs (PhOLEDs) have an Achilles heel, and that is the use of non-sustainable noble metal emitters. Purely organic thermally activated delayed fluorescence (TADF) OLEDs show comparable performance to PhOLEDs and use sustainable materials (Lin et al., 2016). TADF emitters rely mainly on a twisted donor acceptor structure (Chen et al., 2016; Wang et al., 2017). Heterocycles, such as carbazole derivatives (Albrecht et al., 2015), triphenylamines (Data et al., 2016), phenoxazines (Takahashi et al., 2014), and acridines (Zhang et al., 2016), are suitable donors to construct TADF molecules. Electron-accepting units, such as benzonitriles (Park et al., 2016), triazines (Kim et al., 2015), 
benzophenones (Lee et al., 2015), and sulfones (Lee et al., 2016), are commonly used as acceptors.

Three-coordinate boranes have also been reported as electronwithdrawing acceptors in donor-acceptor systems (Numata et al., 2015; Kitamoto et al., 2016; Chen et al., 2017; Lee et al., 2017). However, the high susceptibility of boron-carbon $(\mathrm{B}-\mathrm{C})$ bond cleavage in electron-rich and electron-poor $\mathrm{BN}$ heterocycles along with vacant $\mathrm{p}_{\mathrm{z}}$-orbitals on the boron atom has resulted in poor chemical and photostability (Yang et al., 2016). For example, the $\mathrm{BN}$ heterocycles undergo photoelimination reaction to form a new $\pi$-conjugated polycyclic azaborine compounds (Lu et al., 2013; Yang et al., 2016). In order to increase the chemical and photostability of these materials, steric bulk is added in the vicinity of the boron atom, leading to airstable derivatives. Suzuki and coworkers (Suzuki et al., 2015) synthesized two stable boron-containing compounds based on the bis(mesityl)borane $\left(\mathrm{BMes}_{2}\right)$ acceptor and realized efficient sky-blue [Commission Internationale de l'Eclairage (CIE): 0.18, 0.43 ] and green (CIE: $0.22,0.55$ ) TADF OLEDs with maximum external quantum efficiency $\left(\mathrm{EQE}_{\max }\right)$ of $21.6 \%$ (2DAC-Mes $\left.{ }_{3} \mathrm{~B}\right)$

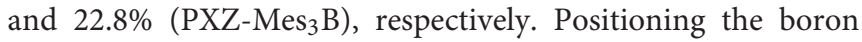
atom within polycyclic $\pi$ systems is another strategy to achieve improved stability of the emitter. With a demethylative direct borylation method, Hatakeyama et al. reported synthesis of stable benzo[fg]tetracenes core containing boronate ester, amide, and thioester substructures. Depending on the heteroatom fragment $(\mathrm{O}$ and $\mathrm{NMe})$ adjacent to the boron, these materials exhibited characteristic emission in the UV at 335 to $377 \mathrm{~nm}$ (Katayama et al., 2016; Numano et al., 2016). Müllen et al. also reported a series of OBO-doped tetrabenzo[bc,ef,kl,no] coronenes and tetrabenzo $[a, f, j, o]$ perylenes, which exhibited structured blue fluorescence with $\phi_{\mathrm{PL}}$ of 61 and 27\%, respectively (Wang et al., 2016). In 2019, Yasuda et al. reported two TADF emitters using boronate ester or boronate thioester as the acceptor (Matsuo and Yasuda, 2019). The boronate thioester-based device showed sky-blue emission at $489 \mathrm{~nm}$ (CIE: $0.17,0.39$ ) and excellent EL performance with the $\mathrm{EQE}_{\max }$ of $20.9 \%$. Although the boronate ester-based device exhibited only the $\mathrm{EQE}_{\max }$ of $5.2 \%$ because of its low PLQY of 28\%, its bluer emission at $471 \mathrm{~nm}$ (CIE: 0.17, 0.22 ) revealed the potential of the boronate ester acceptor for blue and even deep blue TADF emitters.

Herein we report six examples of blue emitters based on this OBO-based benzo[fg]tetracene acceptor (OBO). We systematically investigate the impact of the number, identity of the donor (9,9-dimethyl-9,10-dihydroacridine, DMAC, and $10 \mathrm{H}$-phenoxazine, PXZ), and their position relative to $\mathrm{OBO}$ on the optoelectronic properties of the emitter (Figure 1).

\section{RESULTS AND DISCUSSION}

\section{Synthesis}

These six OBO-based derivatives were successfully synthesized via a three-step procedure (Scheme 1). First, DMAC and PXZ were treated with halo-substituted anisoles under BuchwaldHartwig cross-coupling conditions to afford the corresponding coupled products $1,2,5$, and $\mathbf{6}$ in good to excellent yield. These intermediates were reacted with arylboronic acids/esters under Suzuki-Miyaura cross-coupling conditions to produce dimethoxyteraryl intermediates $(\mathbf{3}, \mathbf{4}, \mathbf{7 - 1 0})$ in good yields. Finally, the target OBO-based emitters were obtained through a demethylative direct borylation procedure of the aryl methyl ethers in the presence of $\mathrm{BBr}_{3}$ (Wang et al., 2016). This final cyclization proceeded in 55 to $56 \%$ yield (DMAC-OBO and PXZ-OBO) when the donor units were introduced to the central phenyl ring. On the other hand, the yields dropped to 21 to $24 \%$ when the donor units were in para position to the methoxy group. The final products were found to be easily purified by silica gel column chromatography and stable under ambient conditions for more than 2 years, showing no decomposition in nuclear magnetic resonance spectra.

The crystals of PXZ-OBO, 5PXZ-OBO, and 5DMACOBO suitable for single crystal X-ray diffraction analysis were obtained from a mixed solution of dichloromethane (DCM) and cyclohexane. The structures reveal that these three molecules possess the expected highly twisted donoracceptor conformation (Figure 2). The dihedral angles between donor and acceptor for the two structural isomers PXZ-OBO and 5PXZ-OBO are tuned remarkably. For PXZ$\mathrm{OBO}$, this torsion is $75.5^{\circ}$, whereas the donor-acceptor dihedral angle is larger at $86.2^{\circ}$ for 5PXZ-OBO. The DMAC analog, 5DMAC-OBO, shows a slightly reduced twisting angle between the donor and the acceptor at $80.1^{\circ}$. In all the structures, the OBO-containing acceptor itself is flat.

\section{Theoretical Calculations}

Density functional theory (DFT) calculations were performed to assess the electronic structure of the six emitters using the PBE0 (Adamo and Barone, 1999) functional and the 6$31 \mathrm{G}(\mathrm{d}, \mathrm{p})$ basis set (Cancès et al., 1997) implemented within Gaussian 09 (Frisch et al., 2009). The ground state geometries of the molecules were optimized in the gas phase starting with the geometry obtained from the single crystal X-ray diffraction analysis. Time-dependent DFT calculations were performed within the Tamm-Dancoff approximation (TDA) on the ground state optimized molecular structures (Hirata and Head-Gordon, 1999). The energies and electron density distributions of the highest occupied and lowest unoccupied molecular orbitals (HOMO/LUMO) and the energies of the $\mathrm{S}_{1}$ and $\mathrm{T}_{1}$ states are shown in Figure 3, and the data are summarized in Tables 1, 2, and Supplementary Table 1, and the electron density distributions of other molecular orbitals are summarized in Supplementary Table 2. As indicated in the SI, the $\mathrm{HOMO} \rightarrow$ LUMO transition is the dominant contribution to the $S_{1}$ state.

The near orthogonal dihedral angle between the donor and acceptor units in the ground state geometries matched with the single crystal data analysis. The HOMO of the emitters is localized on the donor DMAC or PXZ groups, while the LUMO of the emitters is localized on the electron-withdrawing $\mathrm{OBO}$ units. There is minimal spatial overlap between the HOMO and LUMO, which suggests a strong CT character of the excited singlet state and which results in a small calculated $\Delta E_{S T}$ for 


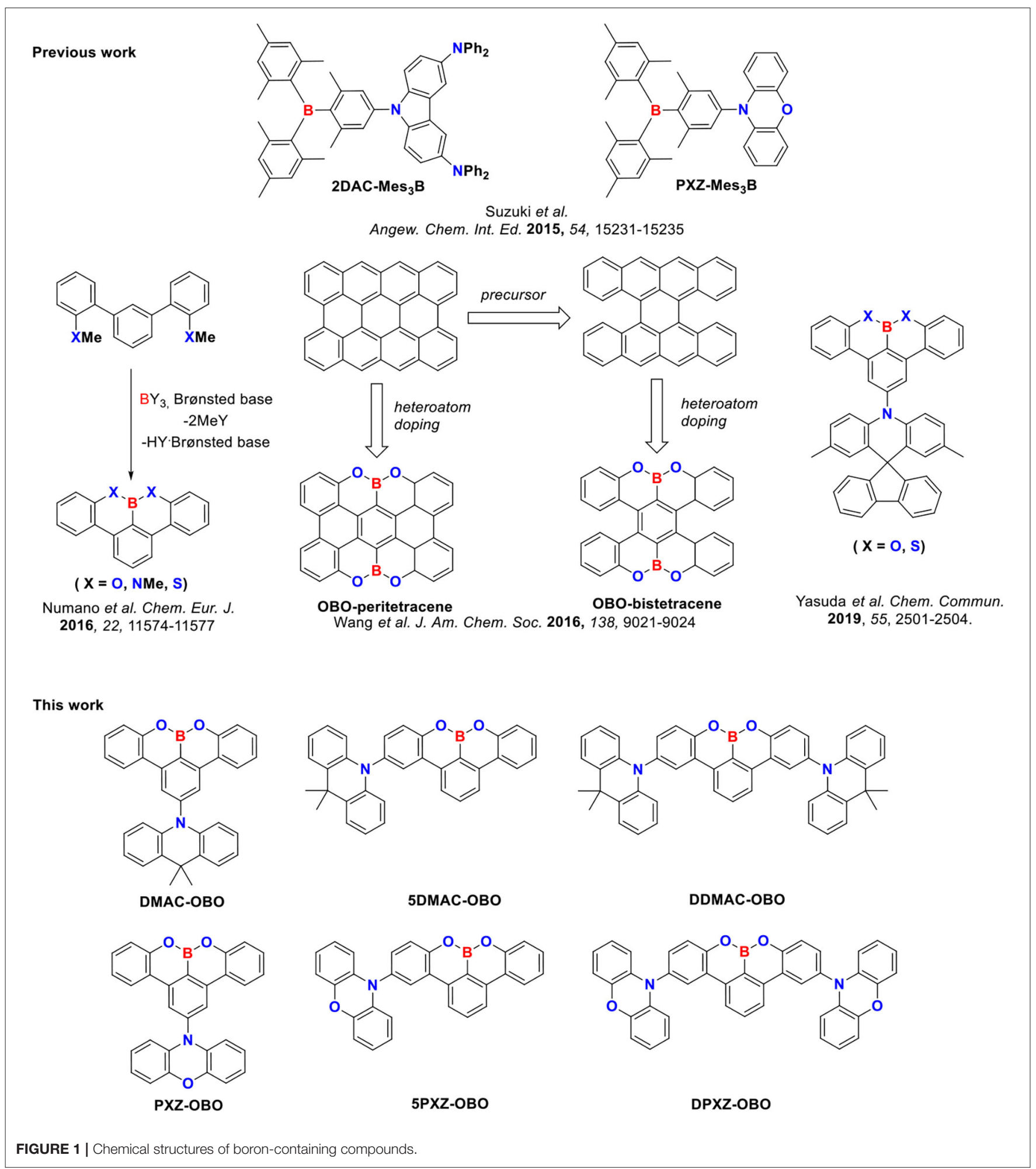

the ground state geometry. The $S_{1}$ energies for the DMAC series ranged narrowly from 3.22 to $3.19 \mathrm{eV}$, showing potential as blue emitters. The use of the stronger PXZ donor results in smaller $\Delta E_{\mathrm{ST}}$ values and lower $\mathrm{S}_{1}$ energies $(2.72-2.83 \mathrm{eV})$ compared to the DMAC analogs; the use of two donor units in DDMAC$\mathrm{OBO}$ and DPXZ-OBO also contributed to decreased $\Delta E_{\mathrm{ST}}$. Even though these values are derived for the vertical transition from the ground state geometry, the calculated $\Delta E_{S T}$ values for all 


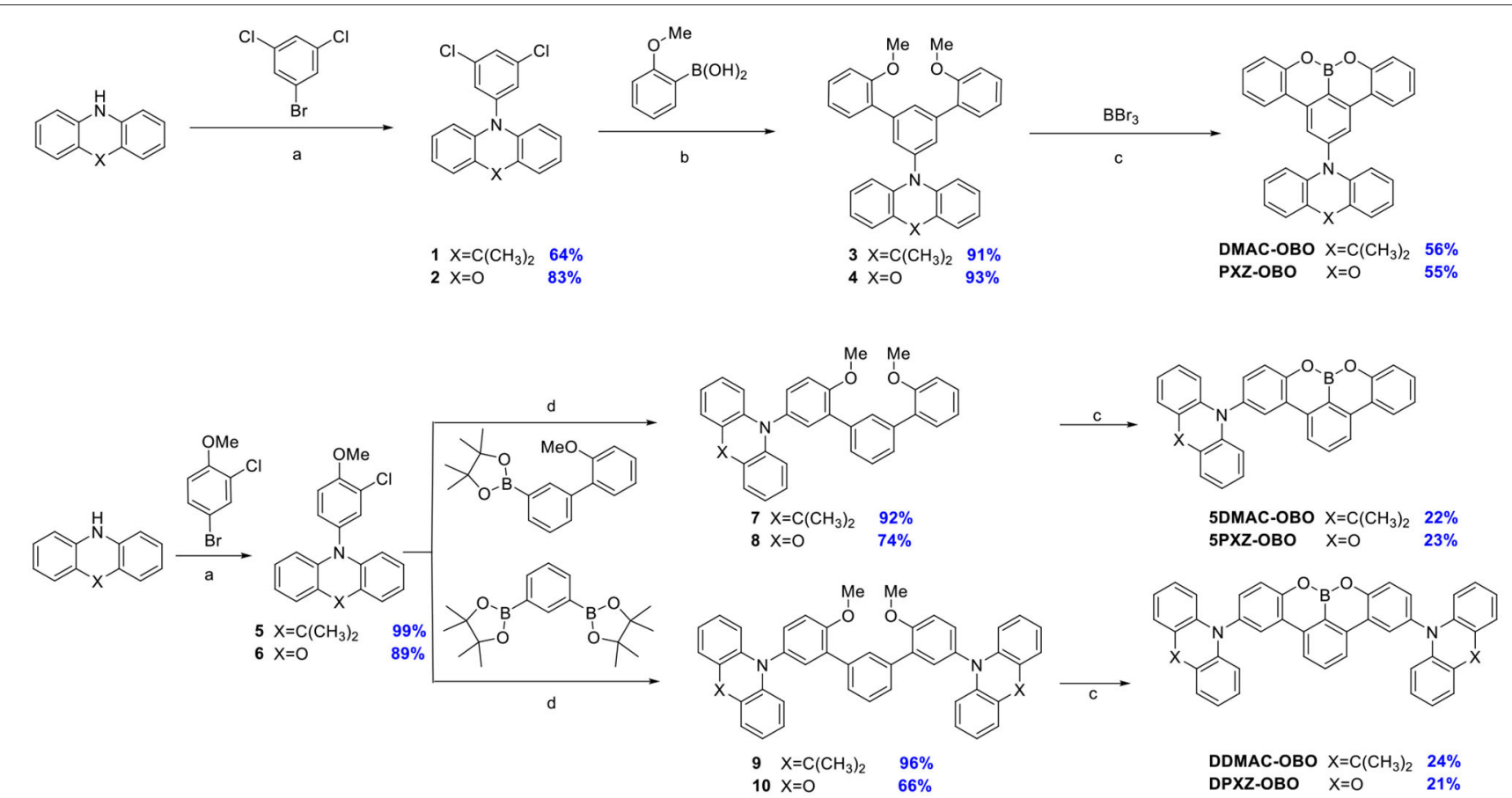

SCHEME 1 | Synthetic route for OBO-based derivatives. a. $\mathrm{Pd}(\mathrm{OAc})_{2}$, xantphos, $\mathrm{NaO}{ }^{\mathrm{B}} \mathrm{Bu}, \mathrm{PhMe}, 100^{\circ} \mathrm{C}, 12 \mathrm{~h}$, argon; b. $\mathrm{Pd}(\mathrm{OAc})_{2}, \mathrm{SPhos} \mathrm{K}_{3} \mathrm{PO} 4, \mathrm{THF} / \mathrm{H}_{2} \mathrm{O}, 80^{\circ} \mathrm{C}$, $12 \mathrm{~h}$; c. $150^{\circ} \mathrm{C}, 1,2$-dichlorobenzene, $12 \mathrm{~h}$, argon; d. $\mathrm{Pd}(\mathrm{OAc})_{2}$, SPhOS, $\mathrm{K}_{3} \mathrm{PO}_{4}, \mathrm{PhMe}, 100^{\circ} \mathrm{C}, 12 \mathrm{~h}$.
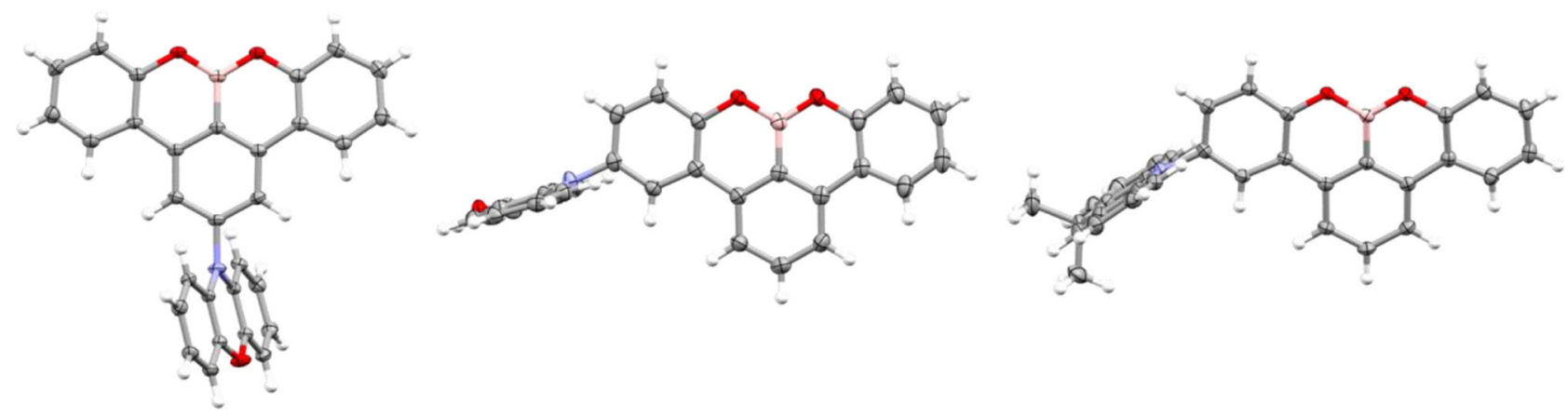

FIGURE 2 | Molecular structure of PXZ-OBO (left, CCDC 1973638), 5PXZ-OBO (middle, CCDC 1973639), and 5DMAC-OBO (right, CCDC 1973640, one of the two crystallographically independent molecules is shown) drawn at $50 \%$ probability level.

emitters are sufficiently small to justify their further evaluation as TADF emitters.

\section{Electrochemical Properties}

The electrochemical properties of the six emitters were examined by cyclic voltammetry (CV) as shown in Figure 4, and the results are listed in Table 1. The reduction wave was assigned to the electron-accepting $\mathrm{OBO}$ unit and found to be irreversible. The LUMO value for DMAC-OBO, 5DMAC-OBO, and DDMAC-OBO were determined to be $-2.69,-2.96$, and $-2.93 \mathrm{~V}$, respectively, from the reduction potentials obtained by CV. For the PXZ analogs PXZ-OBO, 5PXZ-OBO, and DPXZ-OBO, the LUMO values were determined to be -2.70 ,
-2.82 , and $-2.99 \mathrm{~V}$, respectively. The LUMO of 5DMAC$\mathrm{OBO}$ is lowest among DMAC-based emitters, whereas the most stabilized LUMO in the PXZ family of compounds is DPXZ-OBO. The oxidation wave was found to be reversible in all six emitters, which demonstrates that PXZ and DMAC radical cations are electrochemically stable. The DMAC-based emitters show slightly more anodic oxidation potentials than the corresponding PXZ-based emitters, in line with their relative electron-donating ability.

\section{Photophysical Properties}

The newly synthesized $\mathrm{OBO}$ derivatives form two structurally analogous series. While the $\mathrm{OBO}$ moiety 
A

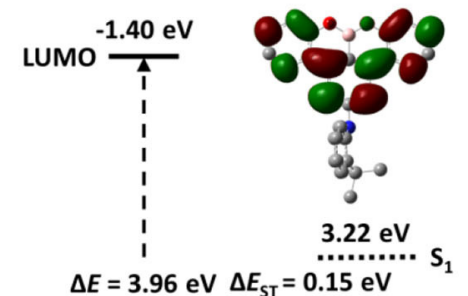

$\Delta E=3.96 \mathrm{eV} \Delta E_{\mathrm{ST}}=0.15 \mathrm{eV} \ldots \ldots \ldots \mathrm{T}_{1}$

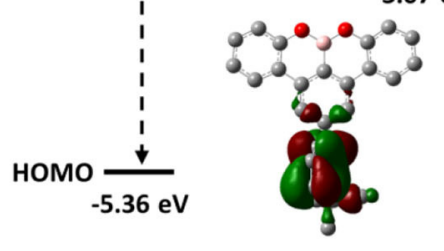

B

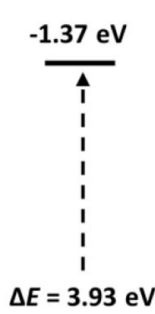

$\Delta E=3.93 \mathrm{eV}$

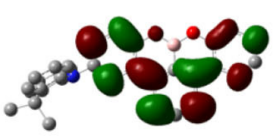

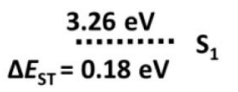
$3.08 \mathrm{eV}$

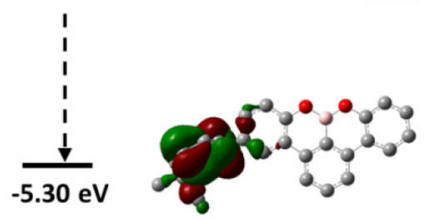

5DMAC-ОВО

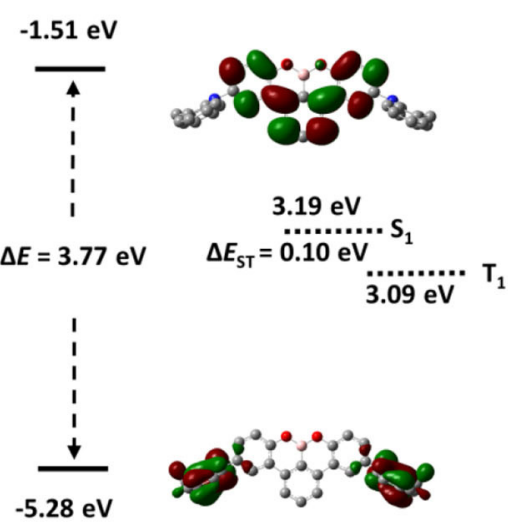

DDMAC-OBO

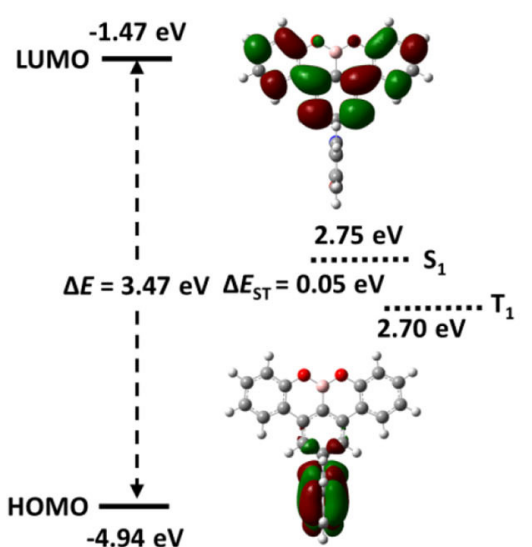

PXZ-Ово
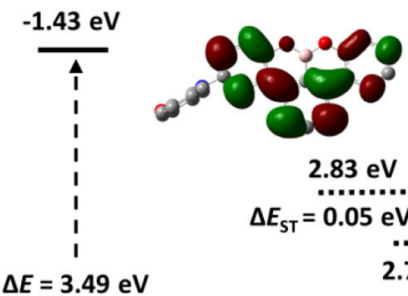

$2.83 \mathrm{eV}$ $\Delta E_{\mathrm{ST}}=0.05 \mathrm{eV} \mathrm{S}_{1}$ …....... $\mathrm{T}_{1}$
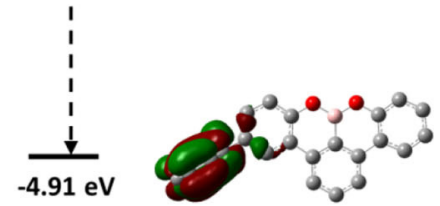

5PXZ-ОВо
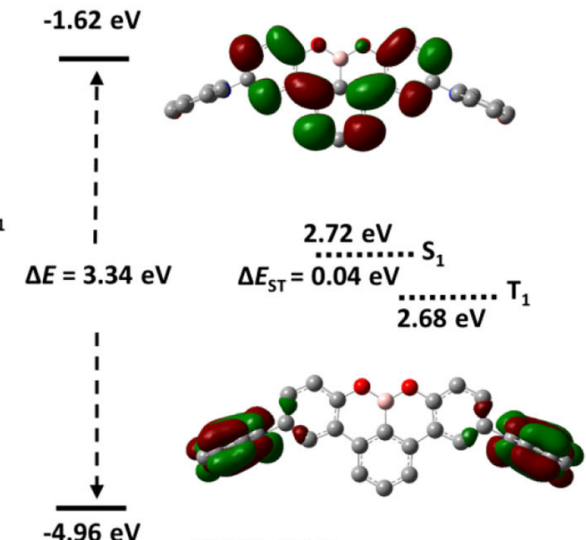

DPXZ-ОBO

FIGURE 3 | DFT calculated ground and excited state energies and electron density distributions of the HOMOs and LUMOs for (A) DMAC-based OBO emitters and (B) PXZ-based OBO emitters. The orbitals are derived using the ground state geometry.

TABLE 1 | Summary of electrochemical and DFT/TDA calculated photophysical properties of OBO-based emitters.

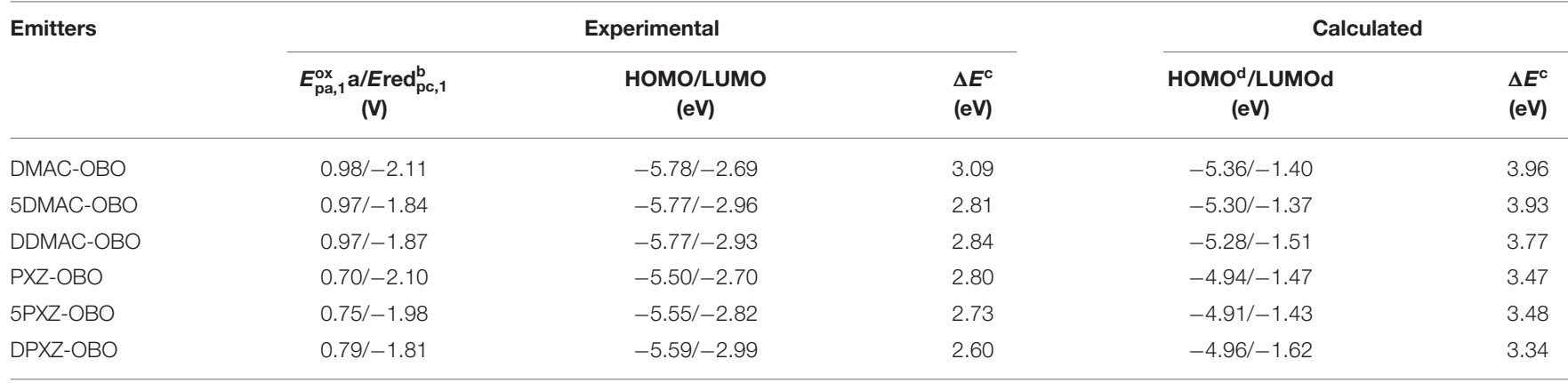

a In $\mathrm{CH}_{2} \mathrm{Cl}_{2}$ and ${ }^{\mathrm{b}}$ in DMF, with $0.1 \mathrm{M}\left[\mathrm{nBu_{4 }}{\mathrm{N}] P F_{6}}_{6}\right.$ as the supporting electrolyte, $\mathrm{Pt}$ as the working electrode, $\mathrm{Ag} / \mathrm{AgCl}$ as the reference electrode and Pt wire as the counter-electrode. $\mathrm{FC}_{\mathrm{C}} \mathrm{FC}^{+}$was used as the internal reference and the data reported vs. SCE. The HOMO and LUMO energies were calculated using the relation $E_{H O M O} / L U M O=-\left(E_{p a, 1}^{O X} / E_{p c, 1}^{\text {red }}+4.8\right) \mathrm{eV}$, where $E_{p a}^{O X}$ and $E_{p c}^{\text {red }}$ are anodic and cathodic peak potentials, respectively. ${ }^{c} \Delta E=\left|E_{H O M O}-E_{L U M O}\right| \cdot{ }^{d}$ Determined from the DFT or TDA-DFT calculations.

always takes the acceptor role, in the first series, DMAC is used as donor moiety, and PXZ is used as donor for the second series. We address here how changing the number and position where the donor is attached impacts on the electronic structure of the TADF molecule. 
TABLE 2 | $\mathrm{S}_{1}$ and $\mathrm{T}_{1}$ energies determined in 2-MeTHF solution At $77 \mathrm{~K}$ and from theoretical calculation.

\begin{tabular}{|c|c|c|c|c|c|c|}
\hline Compounds & $\mathrm{S}_{1}(77 \mathrm{~K})(\mathrm{eV})$ & $\mathrm{S}_{1}^{\text {theor }}(\mathrm{eV})$ & $\mathrm{T}_{1}(77 \mathrm{~K})(\mathrm{eV})$ & $\mathrm{T}_{1}^{\text {theor }}(\mathrm{eV})$ & $\Delta E_{\mathrm{ST}}(77 \mathrm{~K})(\mathrm{eV})$ & $\Delta E_{\mathrm{ST}}^{\text {theor }}(\mathrm{eV})$ \\
\hline DMAC-OBO & 3.43 & 3.22 & 2.69 & 3.07 & 0.74 & 0.15 \\
\hline 5DMAC-OBO & 3.60 & 3.26 & 2.69 & 3.08 & 0.91 & 0.18 \\
\hline PXZ-OBO & 3.34 & 2.75 & 2.70 & 2.70 & 0.64 & 0.05 \\
\hline 5PXZ-OBO & 3.34 & 2.83 & 2.70 & 2.78 & 0.64 & 0.05 \\
\hline
\end{tabular}

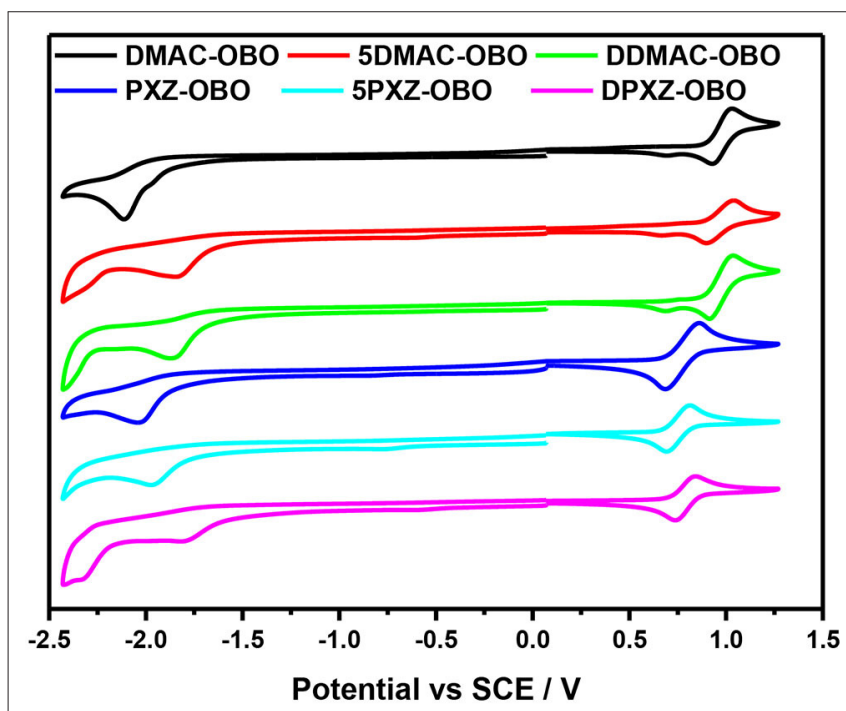

FIGURE 4 | Cyclic voltammograms of OBO-based emitters (reduction and oxidation scans were carried out in degassed dimethylformamide (DMF) and DCM, respectively, at a scan rate $100 \mathrm{mV} \mathrm{s}^{-1}$ ).

\section{Absorption and Emission in Solutions}

The absorption spectra of the emitters in $\mathrm{CHCl}_{3}$ are shown in Figure 5A. In solution, all the emitters exhibit similar absorption profiles with the absorption peaks between 260 and $340 \mathrm{~nm}$. Taking into account the structureless absorption spectra of the donors, one can assign these maxima to localized $\pi$ $\pi^{*}$ transitions of the acceptor OBO moiety (Numano et al., 2016). In the case of DMAC molecules, the absorption of the donor is masked by absorption of the acceptor and cannot be clearly observed (Rodella et al., 2020). In contrast, for the PXZ compounds, the contribution of the donor is distinguished as the shoulder at $350 \mathrm{~nm}$ (Mantsch, 1969). On the semilogarithmic scale, weak broad structureless absorption bands can be seen in the low-energy part of the spectra. These broad bands are ascribed to an intramolecular charge transfer transition that corresponds to transfer of electron from the PXZ or DMAC donor to the $\mathrm{OBO}$ acceptor, in agreement with the theoretical calculations. In the case of the PXZ molecules, this band is shifted to longer wavelengths by $25 \mathrm{~nm}(0.2 \mathrm{eV})$.

The photoluminescence (PL) spectra are broad and unstructured, an indication of emission from a CT state
(Figure 5B). The PL spectra of the DMAC-based emitters in $\mathrm{CHCl}_{3}$ show blue-green emission with maxima $\left(\lambda_{\mathrm{PL}}\right)$ between 448 and $460 \mathrm{~nm}$, whereas for the PXZ-based emitters, a red-shifted emission is observed maxima ranging more narrowly between 497 and $501 \mathrm{~nm}$. The weak bands observed for PXZcontaining molecules can be assigned to emission of the donor (see discussion of Figure 6).

Steady-state (SS) emission in 2-methyltetrahydrofuran (2$\mathrm{MeTHF}$ ) at 300 and $77 \mathrm{~K}$ was investigated to explore the energy of the excited states of the emitters (Figure 6 and Table 2). At $300 \mathrm{~K}$, all molecules demonstrate a broad emission spectrum centered at about $2.6 \mathrm{eV}$ (DMAC-containing compounds) or $2.3 \mathrm{eV}$ (PXZ-containing compounds) that is close to the, respectively, spectrum observed in $\mathrm{CHCl}_{3}$. This band can be assigned to a transition from lowest stabilized CT state. Concomitantly, when exciting the solutions at $300 \mathrm{~nm}$ for the DMAC-containing molecules, a second, high-energy band is observed with a $\lambda_{\mathrm{PL}}$ at $350 \mathrm{~nm}$. For the more symmetric molecules DMAC-OBO and DDMAC-OBO, this band is not structured, while it carries additional pronounced shoulders/peaks for the less symmetric 5DMAC-OBO. The unstructured band fits well in terms of the shape and position to the spectrum of DMAC fluorescence and can therefore be assigned to emission from an LE state on the acridine donor (the emission spectrum of DMAC has been inserted in Figure 6 for DDMAC-OBO, orange line, for ease of reference). The pronounced features in the 5DMAC$\mathrm{OBO}$ exactly match the energies and linewidth reported from Numato et al. at $3.65,3.50,3.35$, and $3.20 \mathrm{eV}$ for the vibronic progression of the emission associated with the OBO-acceptor moiety (Numano et al., 2016). Evidently, for the less symmetric 5DMAC-OBO compound, there is LE emission predominantly from the acceptor, in contrast to the LE emission from only the donor for the two more symmetric compounds. For the PXZ compounds, we also observe the characteristic vibronically wellresolved emission from the $\mathrm{OBO}$ acceptor for the less symmetric $5 \mathrm{PXZ}-\mathrm{OBO}$, whereas it is not present in the more symmetric PXZ-OBO and DPXZ-OBO.

Dual emission is not a common phenomenon in general, as internal conversion (IC) from the higher to the lower excited state usually competes efficiently with radiative decay from the higher state to the ground state, unless there is a large energy gap separating the two emitting state as, e.g., for azulene. However, such dual emission from a CT and an LE state has already previously been observed for donor-acceptor compounds (Acar et al., 2003; Druzhinin et al., 2015). In such 

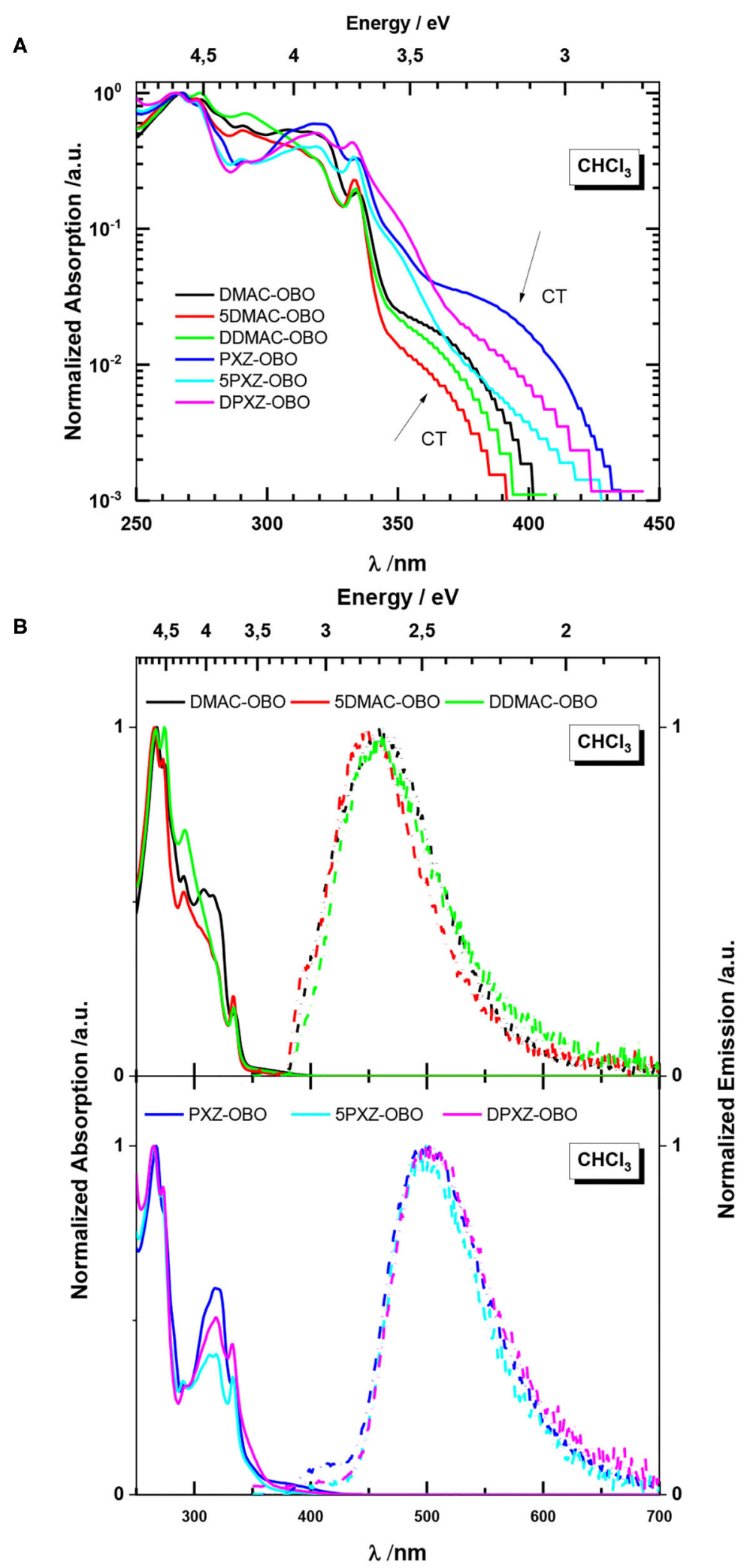

FIGURE 5 | (A) Absorption in $\mathrm{CHCl}_{3}$ on semilogarithmic scale. (B) Absorption (left) and photoluminescence spectra (right) in $\mathrm{CHCl} 3$. ( $\lambda_{\text {exc }}=332 \mathrm{~nm}$ ). 

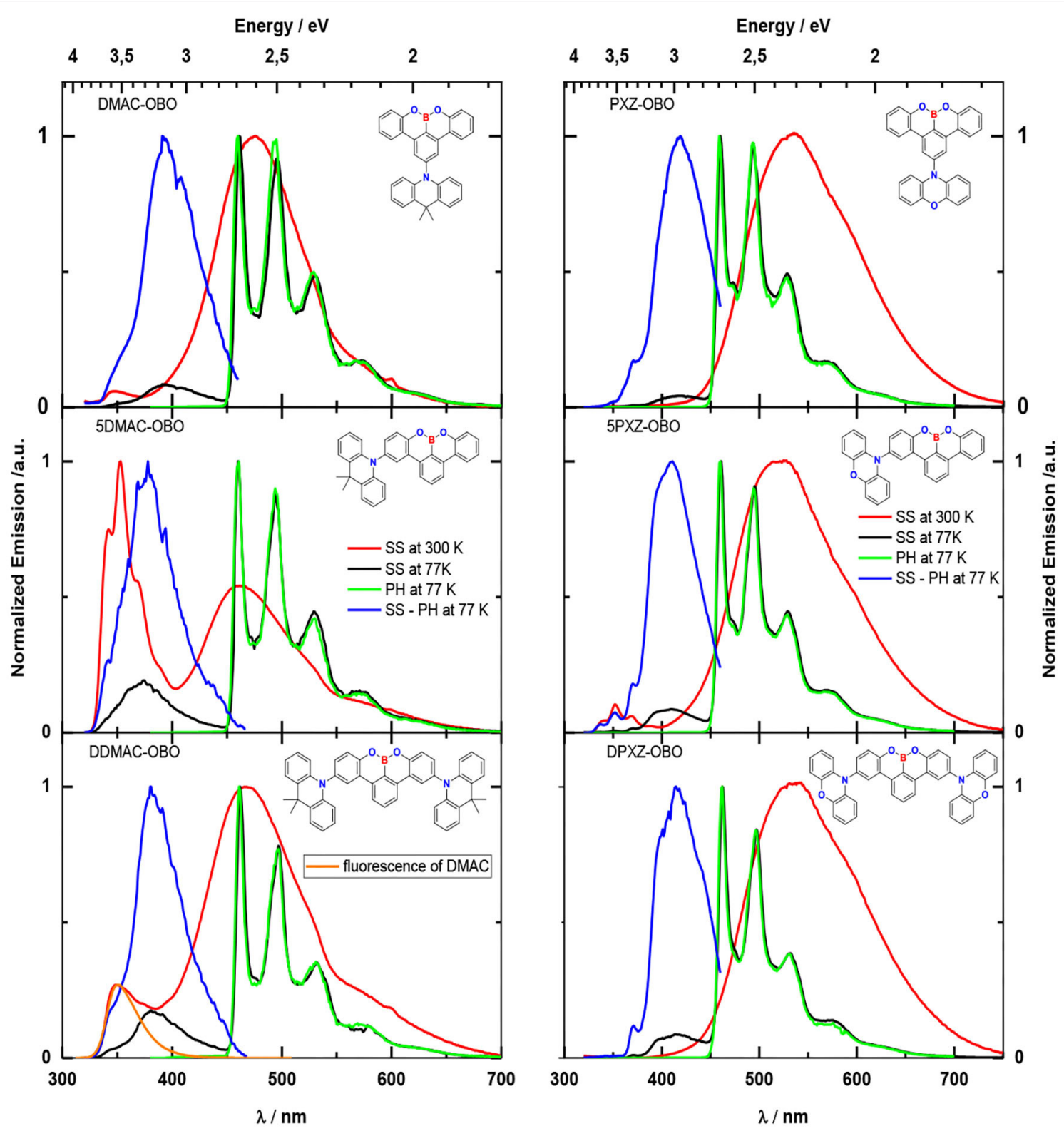

FIGURE 6 | SS emission in 2-MeTHF at $300 \mathrm{~K}$ (red lines), SS emission (black lines), phosphorescence (green lines), fluorescence of OBO-based emitters (blue lines) in 2-MeTHF at $77 \mathrm{~K}$. The fluorescence spectrum was obtained by subtracting the phosphorescence from the SS spectrum and by next normalizing. Excitation was at $300 \mathrm{~nm}$. The concentration was $0.05 \mathrm{mg} / \mathrm{mL}$. The orange line corresponds to DMAC fluorescence at $300 \mathrm{~K}$.

molecules, IC from LE to CT state corresponds to electron transfer between two parts of the molecule. Because of the nearly perpendicular conformation of the donor and acceptor, the initial intramolecular electron transfer can be sufficiently slow so that radiative decay from the LE states may compete with the IC process.

In both series, the intensity of the $\mathrm{CT}$ emission relative to the respective LE emission decreases in the order DMAC-OBO (PXZ-OBO), DDMAC-OBO (DPXZ-OBO), and 5DMAC-OBO (5PXZ-OBO). This order reflects the relative strength of the $\mathrm{y}$-component of the molecular transition dipole moment that would result in a simple picture where the whole density is considered localized on the nitrogen and the electron density on the boron.

The SS PL spectra at $77 \mathrm{~K}$ consist of a strong and highly structured phosphorescence and a weak fluorescence emission. The transition in the SS spectra from 300 to $77 \mathrm{~K}$ leads to a large shift of the fluorescence of the molecules to higher energy (to $3.2 \mathrm{eV}$ for the DMAC compounds and to $3.0 \mathrm{eV}$ for the PXZ compounds). This is caused by the freezing out of the reorientation of solvent shell molecules, so that reorganization of the emitter after excitation is precluded (Lakowicz, 2006). 


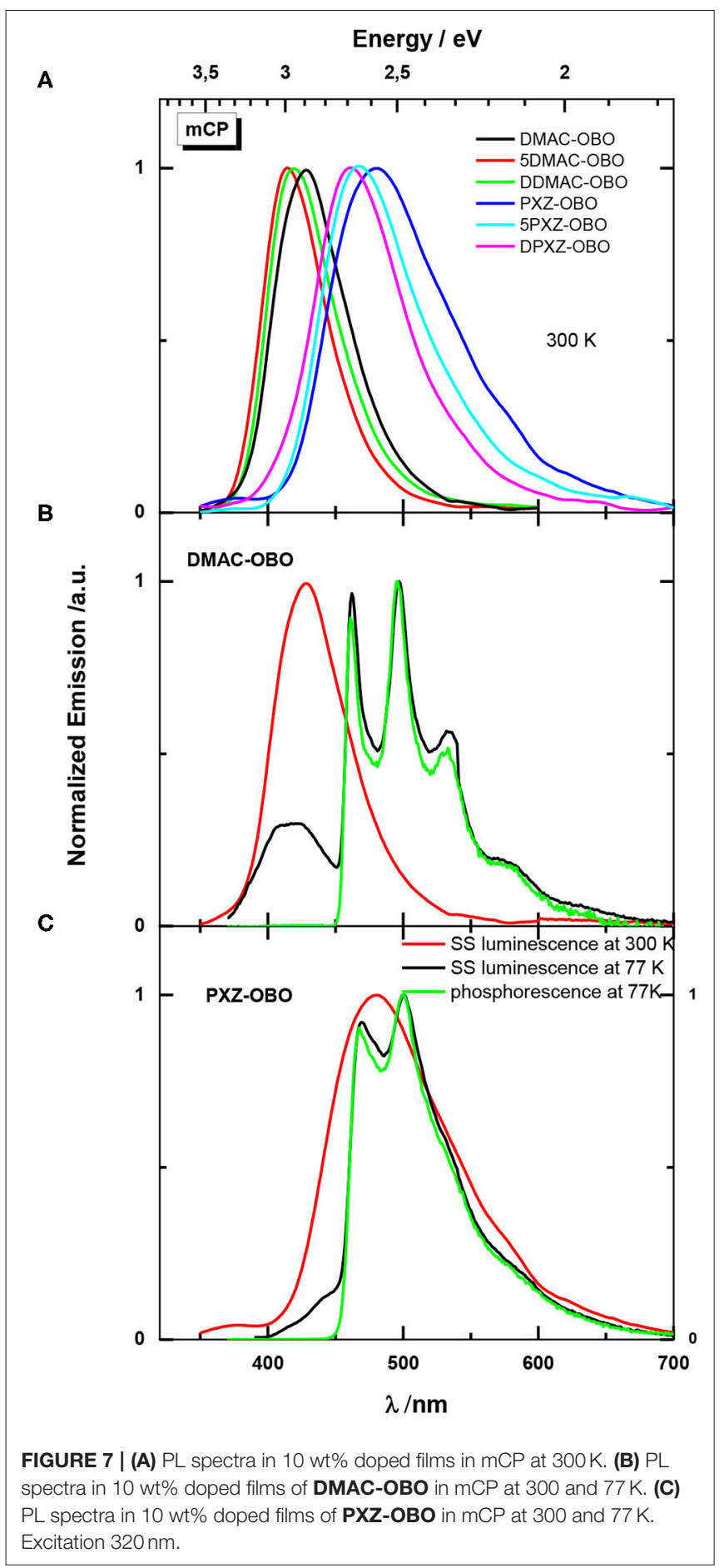

As for $300 \mathrm{~K}$, the $77 \mathrm{~K}$ fluorescence bears the signature of the respective LE states. We point out that the vibrational structure that weakly superimposes on the CT emission for PXZ-OBO and DPXZ-OBO is not that of the OBO acceptor but rather that of the phenoxazine donor LE (Mantsch, 1969), whereas that in 5PXZ-OBO is again the LE state of the OBO acceptor.
The phosphorescence observed has the same position and the same shape for all six molecules, implying its origin is from a purely LE triplet state of the OBO acceptor (Matsuo and Yasuda, 2019). Table 2 presents the values for the singlet and triplet energies and the corresponding $\Delta E_{\mathrm{ST}}$ values. The energy of the triplet and singlet states was obtained from the position of the onsets of the phosphorescence spectra (Figure 6, green lines) and fluorescence spectra (Figure 6, blue lines), respectively. Table 2 shows that the theoretical values for the singlet state energy more or less coincide with experimentally derived values for the DMAC-based molecules, whereas for PXZ-based molecules, the theoretical data are very close the experimental one for the triplet state. Nevertheless, the experimental values for $\Delta E_{S T}(0.64-$ $0.91 \mathrm{eV})$ are significantly higher than the theoretical prediction $(0.04-0.18 \mathrm{eV})$ for both types of molecules, the latter conducted in the gas phase.

\section{Luminescence in Blend mCP Films}

While the solution data are relevant to analyze the excited states of the molecule, for electroluminescence applications the solid-state host materials doped with the emitter need to be considered. So, the PL behavior of these emitters in $\mathrm{mCP}$ films was studied at $300 \mathrm{~K}$ (Figure 7). The DMAC-based derivatives show deep blue emission with $\lambda_{\text {PL }}$ ranging from 415 to $428 \mathrm{~nm}$, whereas the emission of the PXZ-based derivatives is redshifted and blue to sky-blue with $\lambda_{\mathrm{PL}}$ ranging from 458 to $480 \mathrm{~nm}$. Like for measurements in 2-MeTHF glass at $77 \mathrm{~K}$, phosphorescence dominates in emission of the $\mathrm{mCP}$ blend films at $77 \mathrm{~K}$. The phosphorescence of the compounds in the DMAC series coincides with the phosphorescence observed in 2-MeTHF glass, implying that this emission corresponds to acceptor phosphorescence. However, for PXZ-based molecules, the phosphorescence differs in shape and position from measurements in 2-MeTHF glass. In fact, this phosphorescence in the shape and position coincides with phosphorescence of the donor (Mantsch, 1969). This implies that for PXZ-based molecules, there are two LE triplet states that are very close in energy. This is not the case for DMAC-based molecules, where donor triplet state has an energy of around 3.1 Ev (Rodella et al., 2020). The relative position of these lowest triplet states can vary, depending on the host properties. The weak fluorescence at $77 \mathrm{~K}$ is close in position to that observed at $300 \mathrm{~K}$ (Figures 7B,C). The energy of the CT singlet states in the films is lower in comparison with that measured in the 2-MeTHF glass at $77 \mathrm{~K}$. This effect can be explained by considering that dispersion effects are negligible for small-molecular solvents but can be remarkable in the case of adjacent host molecules with an extended $\pi$-electron system as is the case with $\mathrm{mCP}$. Thus, after excitation, the resulting change of dipole moment of the emitter leads to a change of electron distribution in the host molecule, which in turn leads to stabilization of the excited state of the dopant.

\section{Transient Luminescence in mCP Blend Films}

To allow for an analysis of the TADF properties, Figure 8A presents the transient decay of the emission of the molecules under investigation in $10 \mathrm{wt} \% \mathrm{mCP}$ blend films at $300 \mathrm{~K}$. 
A

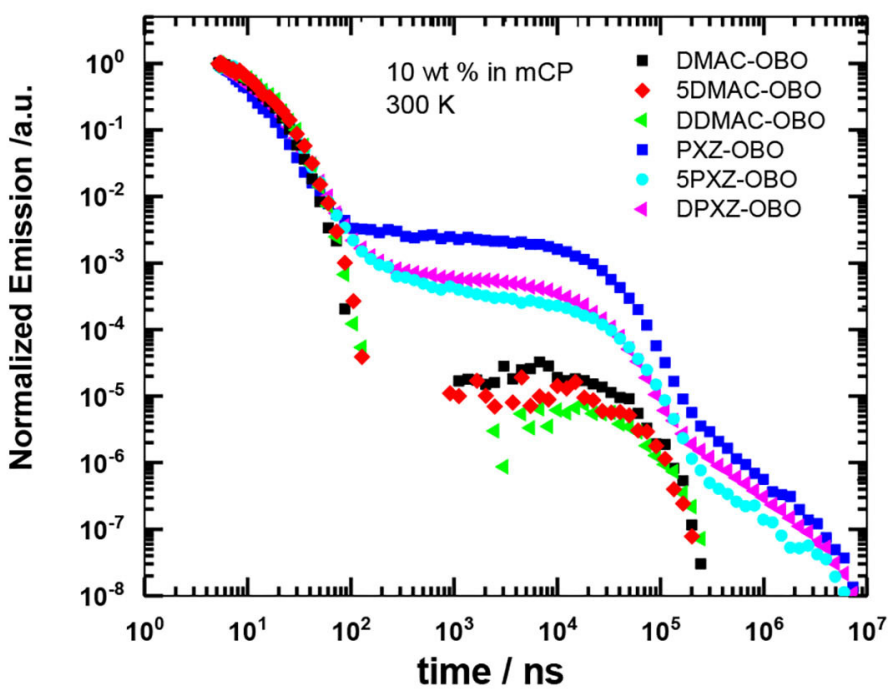

B

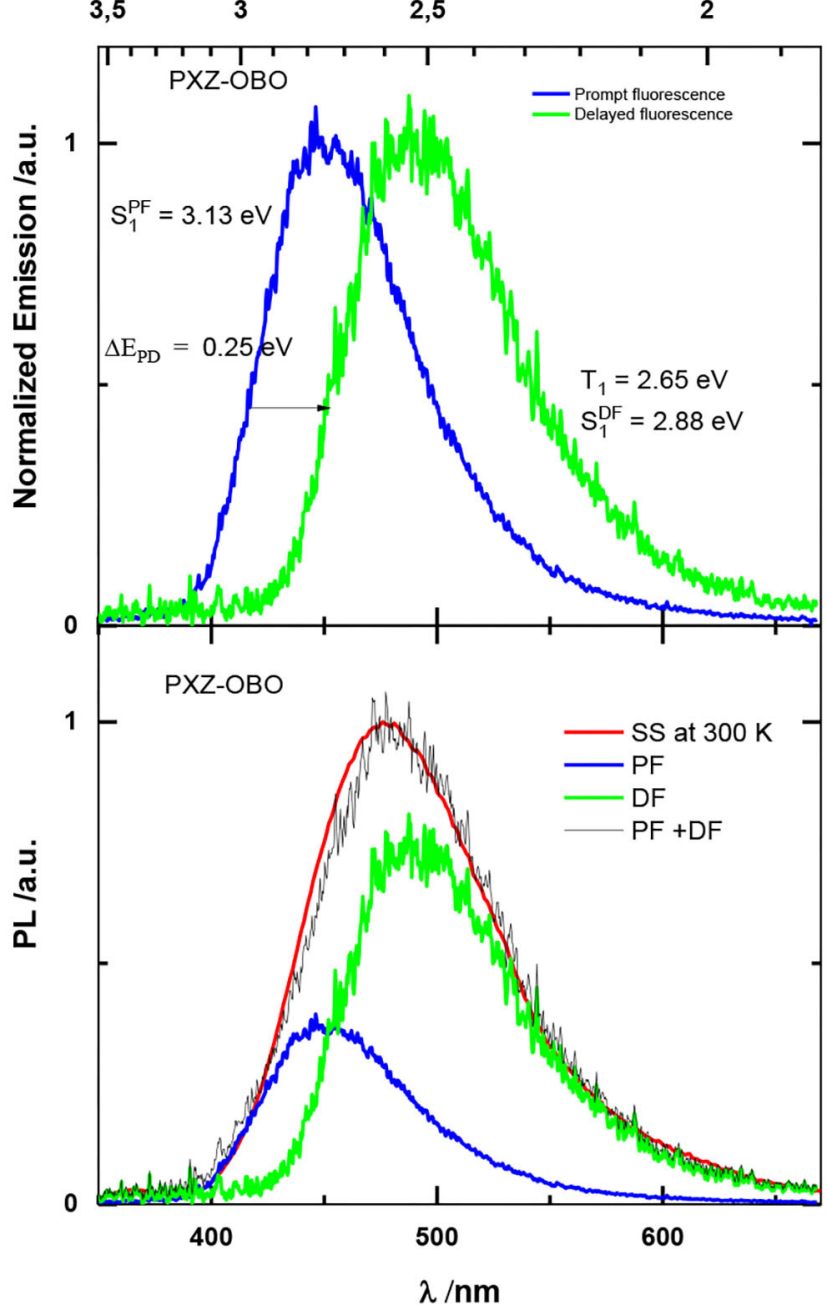

FIGURE 8 | (A) Transient data for blend films in mCP at $300 \mathrm{~K}$. (B) Spectra of prompt (delay $10 \mathrm{~ns}$, gate $10 \mathrm{~ns}$ ) and delayed fluorescence (delay $1 \mu \mathrm{s}$, gate $100 \mathrm{~ns}$ ) of PXZ-OBO in mCP film at $300 \mathrm{~K}$. (C) Analysis of the spectrum of total emission of PXZ-OBO in mCP film using the spectra of prompt and delayed components from (A). 
TABLE 3 | Photophysical properties of OBO-based emitters.

\begin{tabular}{|c|c|c|c|c|c|c|c|c|}
\hline Compounds & $\lambda_{\mathrm{PL}}^{\mathrm{a}}(\mathrm{nm})$ & $\lambda_{\mathrm{PL}}^{\mathrm{b}} / \lambda_{\mathrm{PL}}^{\mathrm{c}}(\mathrm{nm})$ & $\mathrm{FWHM}^{\mathrm{c}}(\mathrm{eV})$ & $\operatorname{CIE}(x, y)^{\mathrm{c}}$ & PLQY $^{b} / P Q^{c}(\%)$ & $\tau_{\mathrm{p}}^{b}(\mathrm{~ns})$ & $\tau_{\mathrm{d}} \mathrm{b}(\mu \mathrm{s})$ & $I_{\mathrm{DF}} / I_{\mathrm{PF}}^{\mathrm{b}}$ \\
\hline DMAC-OBO & 460 & $429 / 446$ & 0.56 & $0.16,0.13$ & $5 / 8$ & 8 & 37 & 0.07 \\
\hline 5DMAC-OBO & 448 & $415 / 410$ & 0.53 & $0.17,0.10$ & $9 / 8$ & 9 & 42 & 0.04 \\
\hline DDMAC-OBO & 461 & $419 / 420$ & 0.55 & $0.16,0.11$ & $8 / 10$ & 10 & 57 & 0.03 \\
\hline PXZ-OBO & 500 & $480 / 491$ & 0.49 & $0.21,0.36$ & $15 / 46$ & 6 & 23 & 4 \\
\hline 5PXZ-OBO & 497 & $466 / 471$ & 0.52 & $0.18,0.24$ & $7 / 19$ & 10 & 30 & 0.6 \\
\hline DPXZ-OBO & 501 & $462 / 481$ & 0.50 & $0.20,0.31$ & $9 / 20$ & 11 & 22 & 1 \\
\hline
\end{tabular}

${ }^{a}$ In $\mathrm{CHCl}_{3}$ at $300 \mathrm{~K} .{ }^{b} / \mathrm{n} 10 \mathrm{wt} \%$ doped films in $\mathrm{mCP}$ at $300 \mathrm{~K} .{ }^{\mathrm{c}}$ In $10 \mathrm{wt} \%$ doped films in PMMA at $300 \mathrm{~K} . \tau_{p}$, prompt lifetime. $\tau_{d}$, delayed lifetime.

Considering the large $\Delta E_{\mathrm{ST}}$ for DMAC-based molecules (Table 2), we do not expect any considerable TADF for these compounds and that is confirmed by the experimental data. For PXZ-based molecules, delayed emission is clearly observed, and its contribution is surprisingly high, with a $I_{\mathrm{DF}} / I_{\mathrm{PF}}$ between 0.6 and 4. To explore whether this DF is due to TADF or TTA, we observed its dependence on the intensity of the exciting laser beam. As it is seen from Supplementary Figure 4, this dependence demonstrates a lineal character with slope value close to 1 that points out to monomolecular nature of this emission.

An analysis of the time-resolved spectra helps to understand the mechanism of TADF in case of such large singlet-triplet gap. Figure 8B shows the spectra of the prompt and delayed fluorescence. The DF spectrum is shifted to lower energy by $0.25 \mathrm{eV}$ relative the $\mathrm{PF}$ spectrum; i.e., it results only from the lower energy part of the DOS. Such a broad DOS distribution can result in solid solutions where there are a number of different conformers of donor-acceptor molecules, each associated with different energies of their excited states (Penfold et al., 2018). Figure 8B demonstrates clearly that only molecules with the lowest singlet state energy (the low energy tail of the DOS) and lower singlet-triplet gap energy $(0.25 \mathrm{eV})$ contribute in TADF. One can see from Figure 8C that the spectrum of the SS emission can be well-fitted by a superposition of the spectra of prompt and delayed fluorescence. It is clear that maximum of the SS spectrum and its intensity is determined by TADF, whereas PF forms high-energy tail of the SS spectrum.

\section{Luminescence in Blend PMMA Films}

Unfortunately, the PLQY values for these six compounds in $\mathrm{mCP}$ films are low, ranging from 5 to $15 \%$. Therefore, PMMA as host materials was also investigated to explore the emission and quantum yield of these emitters in a second solid state host material. In $10 \mathrm{wt} \%$ doped PMMA films (Supplementary Figure 5), the PXZ-based derivatives show skyblue emission with $\lambda_{\mathrm{PL}}$ ranging from 471 to $491 \mathrm{~nm}$, whereas the emission of the DMAC-based derivatives is deep blue with $\lambda_{\mathrm{PL}}$ ranging from 410 to $446 \mathrm{~nm}$. When the donor is decorated on the central phenyl ring of the OBO unit, the emission of PXZ-OBO is $491 \mathrm{~nm}$, whereas it is $481 \mathrm{~nm}$ for 5PXZ-OBO with the donor located at the para position to the methoxy group. The corresponding DMAC derivatives demonstrate a similar trend. This trend can be explained by the mesomeric donation of the donor to the oxygen atoms, thereby strengthening the acceptor. The PLQYs of all three DMAC derivatives remain low, between 8 and $10 \%$. In contrast, the family of PXZ derivatives shows higher PLQYs, from 19 to $46 \%$ (Table 3).

\section{CONCLUSIONS}

We report six blue emitters employing an OBO-fused benzo[fg]tetracene core as an acceptor. Experimental studies demonstrate their blue and deep blue emission with $\lambda_{\mathrm{PL}}$ of 415 to $480 \mathrm{~nm}$ in mCP films. Although the DMAC derivatives show the negligible delayed emission $\left(I_{\mathrm{DF}} / I_{\mathrm{PF}}, 0.03-0.07\right)$, the PXZ-based emitters exhibit much higher ratio of delayed emission $\left(I_{\mathrm{DF}} / I_{\mathrm{PF}}, 0.6-4\right)$ with the PLQYs from 5 to $15 \%$. When doped in PMMA, these fluorescent emitters show higher PLQYs, from 19 to 46\%. Current efforts are focused on designing new OBO-based molecules through exploring different donors and novel connectivity strategies between donor and $\mathrm{OBO}$ acceptor to access more promising blue TADF emitters for OLEDs.

\section{DATA AVAILABILITY STATEMENT}

The datasets presented in this study can be found in online repositories. The names of the repository/repositories and accession number(s) can be found in the article/Supplementary Material. The research data supporting this publication can be accessed at https://doi.org/10.17630/ 5acd95b7-73bf-4e5a-abe6-ec23c9120835.

\section{AUTHOR CONTRIBUTIONS}

$\mathrm{ZZ}$ conceived the concept and synthesized and characterized the materials. SK performed the DFT calculations electrochemical measurements. SBa performed the photophysical measurements and, together with AK, wrote the photophysics section. ES, FH, and $\mathrm{ZH}$ participated in results discussions. $\mathrm{MN}$ did the $\mathrm{X}$-ray analyses. ZZ, SK, and EZ-C wrote the manuscript. SBr, AK, and EZ-C supervised the project. All authors contributed to the article and approved the submitted version. 


\section{FUNDING}

This project was supported by the Helmholtz Association Program at the Karlsruhe Institute of Technology. The German Research Foundation (formally Deutsche Forschungsgemeinschaft DFG) in the framework of SFB1176 Cooperative Research Centre Molecular Structuring of Soft Matter (CRC1176, A4, B3, C2, C6) and the cluster 3D Matter Made To Order funded under Germany's Excellence Strategy (3DMM2O EXC-2082/1-390761711) are greatly acknowledged for financial contributions. ZZ acknowledges the financial support from Chinese Scholarship Council (CSC) for his Ph.D. studies. SK acknowledges the financial support from European Union's Horizon 2020 research and innovation programme

\section{REFERENCES}

Acar, N., Kurzawa, J., Fritz, N., Stockmann, A., Roman, C., Schneider, S., et al. (2003). Phenothiazine-pyrene dyads: photoinduced charge separation and structural relaxation in the CT state. J. Phys. Chem. 107, 9530-9541. doi: $10.1021 /$ jp036250u

Adamo, C., and Barone, V. (1999). Toward reliable density functional methods without adjustable parameters: the PBE0 model. J. Chem. Phys. 110, 6158-6170. doi: $10.1063 / 1.478522$

Albrecht, K., Matsuoka, K., Fujita, K., and Yamamoto, K. (2015). Carbazole dendrimers as solution-processable thermally activated delayed-fluorescence materials. Angew. Chem. Int. Ed. 54, 5677-5682. doi: 10.1002/anie. 201500203

Cancès, E., Mennucci, B., and Tomasi, J. (1997). A new integral equation formalism for the polarizable continuum model: Theoretical background and applications to isotropic and anisotropic dielectrics. J. Chem. Phys. 107, 3032-3041. doi: 10.1063/1.474659

Chen, D., Liu, K., Gan, L., Liu, M., Gao, K., Xie, G., et al. (2016). Modulation of exciton generation in organic active planar pn heterojunction: toward low driving voltage and high-efficiency OLEDs employing conventional and thermally activated delayed fluorescent emitters. Adv. Mater. 28, 6758-6765. doi: 10.1002/adma.201600612

Chen, X. L., Jia, J. H., Yu, R., Liao, J. Z., Yang, M. X., and Lu, C. Z. (2017). Combining charge-transfer pathways to achieve unique thermally activated delayed fluorescence emitters for high-performance solutionprocessed, non-doped blue OLEDs. Angew. Chem. Int. Ed. 56, 15006-15009. doi: 10.1002/anie.201709125

Data, P., Pander, P., Okazaki, M., Takeda, Y., Minakata, S., and Monkman, A. P. (2016). Dibenzoa,jphenazine-cored donor-acceptor-donor compounds as green-to-red/NIR thermally activated delayed fluorescence organic light emitters. Angew. Chem. Int. Ed. 55, 5739-5744. doi: 10.1002/anie.201600113

Druzhinin, S. I., Galievsky, V. A., Demeter, A., Kovalenko, S. A., Senyushkina, T., Dubbaka, S. R., et al. (2015). Two-state intramolecular charge transfer (ICT) with 3,5-dimethyl-4-(dimethylamino)benzonitrile (MMD) and its metaIsomer mMMD. ground state amino twist not essential for ICT. J. Phys. Chem. A 119, 11820-11836. doi: 10.1021/acs.jpca.5b09368

Endo, A., Sato, K., Yoshimura, K., Kai, T., Kawada, A., Miyazaki, H., et al. (2011). Efficient up-conversion of triplet excitons into a singlet state and its application for organic light emitting diodes. Appl. Phys. Lett. 98:083302. doi: $10.1063 / 1.3558906$

Frisch, M. J., Trucks, G. W., Schlegel, H. B., Scuseria, G. E., Robb, M. A., Cheeseman, J. R., et al. (2009). Gaussian Rev. D.01. Wallingford, CT : Gaussian, Inc.

Hirata, S., and Head-Gordon, M. (1999). Time-dependent density functional theory within the Tamm-Dancoff approximation. Chem. Phys. Lett. 314, 291-299. doi: 10.1016/S0009-2614(99)01149-5

Katayama, T., Nakatsuka, S., Hirai, H., Yasuda, N., Kumar, J., Kawai, T., et al. (2016). Two-step synthesis of boron-fused double helicenes. J. Am. Chem. Soc. 138, 5210-5213. doi: 10.1021/jacs.6b01674 under Marie Skłodowska Curie Individual Fellowship (MCIF; Agreement No. 748430-THF-OLED). SBa acknowledges support from the Bayrisches Staatsministerium für Wissenschaft und Kunst (Stmwk) in the framework of the initiative SolTech as well as from the German Science foundation (DFG) (no. 392306670). EZ-C and AK acknowledge support from the European Union's Horizon 2020 research and innovation programme under the ITN TADFlife (GA 812872).

\section{SUPPLEMENTARY MATERIAL}

The Supplementary Material for this article can be found online at: https://www.frontiersin.org/articles/10.3389/fchem. 2020.563411/full\#supplementary-material

Kim, M., Jeon, S. K., Hwang, S. H., and Lee, J. Y. (2015). Stable blue thermally activated delayed fluorescent organic light-emitting diodes with three times longer lifetime than phosphorescent organic light-emitting diodes. Adv. Mater. 27, 2515-2520. doi: 10.1002/adma.201500267

Kitamoto, Y., Namikawa, T., Suzuki, T., Miyata, Y., Kita, H., Sato, T., et al. (2016). Dimesitylarylborane-based luminescent emitters exhibiting highly efficient thermally activated delayed fluorescence for organic light emitting diodes. Org. Electron. 34, 208-217. doi: 10.1016/j.orgel.2016.04.030

Lakowicz, J. R. (2006). Principles of Fluorescence Spectroscopy. New York, NY: Springer.

Lee, I. H., Song, W., and Lee, J. Y. (2016). Aggregation-induced emission type thermally activated delayed fluorescent materials for high efficiency in non-doped organic light-emitting diodes. Org. Electron. 29, 22-26. doi: 10.1016/j.orgel.2015.11.019

Lee, S. Y., Yasuda, T., Park, I. S., and Adachi, C. (2015). X-shaped benzoylbenzophenone derivatives with crossed donors and acceptors for highly efficient thermally activated delayed fluorescence. Dalton Trans. 44, 8356-8359. doi: 10.1039/C4DT03608E

Lee, Y. H., Park, S., Oh, J., Shin, J. W., Jung, J., Yoo, S., et al. (2017). Rigidityinduced delayed fluorescence by ortho donor-appended triarylboron compounds: record-high efficiency in pure blue fluorescent organic light-emitting diodes. ACS Appl. Mater. Interfaces 9, 24035-24042. doi: 10.1021/acsami.7b05615

Lin, T. A., Chatterjee, T., Tsai, W. L., Lee, W. K., Wu, M. J., Jiao, M., et al. (2016) Sky-blue organic light emitting diode with $37 \%$ external quantum efficiency using thermally activated delayed fluorescence from spiroacridine-triazine hybrid. Adv. Mater. 28, 6976-6983. doi: 10.1002/adma.201601675

Lu, J.-S., Ko, S.-B., Walters, N. R., Kang, Y., Sauriol, F., and Wang, S. (2013). Formation of azaborines by photoelimination of B,N-heterocyclic compounds. Angew. Chem. Int. Ed. 52, 4544-4548. doi: 10.1002/anie.201300873

Mantsch, H. H. (1969). $\pi$-Electronic structure and reactivity of phenoxazine (1), phenothiazine (2), and phenoxthiin (3). Can. J. Chem. 47, 3173-3178. doi: 10.1139/v69-523

Matsuo, K., and Yasuda, T. (2019). Boronate- and borinate-based $\pi$-systems for blue thermally activated delayed fluorescence materials. Chem. Commun. 55, 2501-2504. doi: 10.1039/C8CC10282A

Numano, M., Nagami, N., Nakatsuka, S., Katayama, T., Nakajima, K., Tatsumi, S., et al. (2016). Synthesis of boronate-based benzofgtetracene and benzohihexacene via demethylative direct borylation. Chem. Eur. J. 22, 11574-11577. doi: 10.1002/chem.201602753

Numata, M., Yasuda, T., and Adachi, C. (2015). High efficiency pure blue thermally activated delayed fluorescence molecules having $1 \mathrm{H}$-phenoxaborin and acridan units. Chem. Commun. 51, 9443-9446. doi: 10.1039/C5CC00307E

Park, I. S., Lee, S. Y., Adachi, C., and Yasuda, T. (2016). Full-color delayed fluorescence materials based on wedge-shaped phthalonitriles and dicyanopyrazines: systematic design, tunable photophysical properties, and OLED performance. Adv. Funct. Mater. 26, 1813-1821. doi: 10.1002/adfm.201505106 
Penfold, T. J., Dias, F. B., and Monkman, A. P. (2018). The theory of thermally activated delayed fluorescence for organic light emitting diodes. Chem. Commun. 54, 3926-3935. doi: 10.1039/C7CC09612G

Rodella, F., Bagnich, S., Duda, E., Meier, T., Kahle, J., Athanasopoulos, S., et al. (2020). High triplet energy host materials for blue TADF OLEDs-a tool box approach. Front. Chem. 8:657. doi: 10.3389/fchem.2020.00657

Suzuki, K., Kubo, S., Shizu, K., Fukushima, T., Wakamiya, A., Murata, Y., et al. (2015). Triarylboron-based fluorescent organic light-emitting diodes with external quantum efficiencies exceeding 20\%. Angew. Chem. Int. Ed. 54, 15231-15235. doi: 10.1002/anie.201508270

Takahashi, T., Shizu, K., Yasuda, T., Togashi, K., and Adachi, C. (2014). Donor-acceptor-structured 1,4-diazatriphenylene derivatives exhibiting thermally activated delayed fluorescence: design and synthesis, photophysical properties and OLED characteristics. Sci. Technol. Adv. Mater. 15:034202. doi: 10.1088/1468-6996/15/3/034202

Tao, Y., Yuan, K., Chen, T., Xu, P., Li, H., Chen, R., et al. (2014). Thermally activated delayed fluorescence materials towards the breakthrough of organoelectronics. Adv. Mater. 26, 7931-7958. doi: 10.1002/adma.2014 02532

Uoyama, H., Goushi, K., Shizu, K., Nomura, H., and Adachi, C. (2012). Highly efficient organic light-emitting diodes from delayed fluorescence. Nature 492, 234-238. doi: 10.1038/nature11687

Wang, K., Liu, W., Zheng, C.-J., Shi, Y.-Z., Liang, K., Zhang, M., et al. (2017). A comparative study of carbazole-based thermally activated delayed fluorescence emitters with different steric hindrance. J. Mater. Chem. C 5, 4797-4803. doi: 10.1039/C7TC00681K

Wang, X. Y., Narita, A., Zhang, W., Feng, X., and Mullen, K. (2016). Synthesis of stable nanographenes with OBO-doped zigzag edges based on tandem demethylation-electrophilic borylation. J. Am. Chem. Soc. 138, 9021-9024 doi: 10.1021/jacs.6b04092

Wong, M. Y., and Zysman-Colman, E. (2017). Purely organic thermally activated delayed fluorescence materials for organic light-emitting diodes. Adv. Mater. 29:160544. doi: 10.1002/adma.201605444

Yang, D.-T., Mellerup, S. K., Peng, J.-B., Wang, X., Li, Q.-S., and Wang, S. (2016). Substituent directed phototransformations of bn-heterocycles: elimination vs isomerization via selective B-C bond cleavage. J. Am. Chem. Soc. 138, 11513-11516. doi: 10.1021/jacs.b07899

Yang, Z., Mao, Z., Xie, Z., Zhang, Y., Liu, S., Zhao, J., et al. (2017). Recent advances in organic thermally activated delayed fluorescence materials. Chem. Soc. Rev. 46, 915-1016. doi: 10.1039/C6CS00368K

Zhang, Y., Zhang, D., Cai, M., Li, Y., Zhang, D., Qiu, Y., et al. (2016). Towards highly efficient red thermally activated delayed fluorescence materials by the control of intra-molecular $\pi-\pi$ stacking interactions. Nanotechnology 27:094001. doi: 10.1088/0957-4484/27/9/094001

Conflict of Interest: The authors declare that the research was conducted in the absence of any commercial or financial relationships that could be construed as a potential conflict of interest.

Copyright (c) 2020 Zhang, Kumar, Bagnich, Spuling, Hundemer, Nieger, Hassan, Köhler, Zysman-Colman and Bräse. This is an open-access article distributed under the terms of the Creative Commons Attribution License (CC BY). The use, distribution or reproduction in other forums is permitted, provided the original author(s) and the copyright owner(s) are credited and that the original publication in this journal is cited, in accordance with accepted academic practice. No use, distribution or reproduction is permitted which does not comply with these terms. 